Review Article

\title{
Status of Quantum Chemistry Research in India
}

\author{
SOURAV PAL ${ }^{1, *}$ and SWAPAN K GHOSH ${ }^{2}$ \\ ${ }^{1}$ Indian Institute of Science Education and Research Kolkata, Mohanpur 741 246, Nadia, West Bengal, \\ India \\ ${ }^{2}$ Centre for Excellence in Basic Sciences, Vidyanagari Campus, University of Mumbai, Kalina, Santacruz \\ (East), Mumbai 400 098, India
}

(Received on 03 March 2019; Revised on 25 May 2019; Accepted on 05 June 2019)

\begin{abstract}
In this article, we present the status of present research in quantum chemistry in India. We note that in early years, there was a significant amount of research dealing with the formalismaspects of quantum chemistry, some of which stand out by global standards. Subsequently, there has been a significant growth in computational quantum chemistry using both ab initio and density functional theories. In this article, we have presented glimpses of the broad nature of significant work done with some important references and by no means, it is supposed to be exhaustive in nature.
\end{abstract}

Keywords: Quantum Chemistry; Quantum Mechanics; Hamiltonian

\section{Introduction}

\section{A Historical Perspective}

As quantum mechanics began to evolve in the beginning of the last century, its impact on chemical sciences began to be felt. Atomic and molecular insights into chemical bonding, reaction, electronic structure and dynamics began to emerge. Initial atomic theory such as Bohr's model was based on ad hoc quantization. $\mathrm{G} \mathrm{N}$ Lewis developed dot structures based on which the octet rule was established even without using formal quantum mechanics. Early work leading to the formulation of quantum mechanics started appearing. Wave-particle duality, Heisenberg's uncertainty principle, Bohr's correspondence principle etc can be cited as examples. Schroedinger's paper appearing in Physical Review in 1926 (Schroedinger, 1926) is quoted widely as the first paper on quantum mechanics. Interestingly, within one year, Heitler and London's paper (Heitler and London, 1927) on bonding in hydrogen molecule appeared based on which Pauling developed the Valence-bond (VB) method( Pauling, 1931). Within a few years, molecular orbital theory based on electrons in orthonormal molecular orbitals (MO), started emerging (James and Coolidge,
1933). This became very attractive for use in large molecules. Eventually MO theory outlived VB theory. Early focus in chemistry was on solution of electronic structure theory and solution of the eigen-value equation of the Hamiltonian. As it became clear that exact results even for two-electron systems are nearly impossible, focus was concentrated on developing approximations based on physical understanding and mathematical simplicity. Along with Hartree-Fock, several electron correlation (Lowdin, 1955) methods started emerging. Perturbation theory and configuration interactions were the early theories to emerge. In 1960's, very accurate coupled-cluster theory was formulated (Cizek, 1966) and this eventually became the gold standard of quantum chemistry. Various different levels of approximation, including multi-configuration based methods evolved accounting for, satisfactorily, what is known as static correlation in modern language.

Along with the methods or approximations as above which were developed for the solution of eigenfunctions and eigen-values of the Hamiltonian (which we will call wave-function based methods), alternative methods based on electron density emerged. In mid 1960's a formal density based

*Authorfor Correspondence: E-mail: snatarajan@iisc.ac.in 
method, called density functional theory (DFT) was formulated. The basic variable in DFT is the electron density, a function in three dimensional (3-D) space, instead of the many-electron wavefunction in configuration space, thus introducing conceptual simplicity and computational economy. Thus, DFT is a density based quantum mechanics, that aims at bypassing the wavefunction and developing quantum mechanics in 3-D space. Although DFT, in its primitive form, started with the Thomas-Fermi theory as early as 1927 ( Thomas, 1927 and Fermi, 1927), soon after the wave mechanics was proposed in 1926, it was elevated to the status of a formal rigorous theory only in 1964, through the pioneering work of Hohenberg, Kohn and Sham (Hohenberg and Kohn, 1964; Kohn and Sham, 1965). The well-known Kohn-Sham (KS) theory of DFT was preceded by the so called HartreeFock Slater theory (Slater, 1972) which was in the same spirit but introduced as a variant of the wavefunction theory by introducing approximations. Applications of the KS version of DFT in band structure calculations of solids also started being pursued more extensively around the same time and today it is the most widely used method in this field of research. As far as electronic structure theory for molecular systems is concerned, wave-function based and DFT based methods started progressing in parallel.

\section{Indian Context}

India was also active in the development and use of quantum chemistry. Early work from India emerged in 1950's from the group of Professor Sadhan Basu, who was known for his elucidation of the quantum mechanical model of Robert S Mulliken. After being trained in Indiana University, he actively pursued quantum chemistry in India. He published six papers on free electron molecular orbital calculations (Sen and Basu, 1968). His studies were primarily in the fields of charge transfer interactions, ligand field spectra, hydrogen bonding, quantum chemistry and photochemistry. He calculated the transition energies and oscillator strengths of aromatic polyhydrocarbons and demonstrated that extended catacondensed planar structures could be derived only by using 3-4-6 membered rings. By employing Hartree-FockBogolyubov method, he illustrated that, unlike triplet transitions, the longest wavelength singlet transitions in linear polyenes converge to a limit [11]. He may be said to be the first quantum chemist in India.

Some years later, overlapping with much of the career of Professor Sadhan Basu, Professor P T Narasimhan became one of the pioneers of computational chemistry in India and was known for his studies on quantum-mechanical interpretation of magnetic resonance data (Sen and Narasimhan, 1975). Narasimhan's contributions were on the theory of nuclear spin coupling constants. He was deeply influenced by Martin Karplus, his mentor. His studies on the structure and properties of molecules were based on quantum-mechanical interpretation of magnetic resonance data and those studies assisted in widening the understanding of chemical bonding, conformation, chemical reactivity and electrical and magnetic properties of molecules. His contributions helped in the development of dynamic nuclear polarization at X-band in India, covering both the instrumentation and chemical applications.

During the 1970's, a few individuals started their careers in quantum chemistry. Some of them were trained abroad and quite a few were from the above groups of Professor Basu and Professor Narasimhan. Professor Mihir Chowdhury, who was a student of Professor Basu, was himself an experimentalist, but encouraged the growth of theory quite significantly. Many of his students took to research in quantum chemistry quite actively. In fact, the school of quantum chemistry in Calcutta (now known as Kolkata) was among the first active schools with quite a few taking to serious quantum chemistry research. In 1970 s, quantum chemistry started taking root in India. Among the eminent practitioners of theory in that period was Professor B M Deb, who was trained abroad as a student of Prof Charles Coulson and started working on aspects of chemical bonding based on density related methods. His group made early developments of DFT in India. A coverage of his work will appear later during the discussion of DFT methods. Professor Debashis Mukherjee, student of Professor Chowdhury, started to make pioneering contributions in mid 1970s on what was a front line area of research globally. His work was based on multi-reference based methods using coupled-cluster wave functions. In particular, Fock space coupled cluster (FSCC) methods (Mukherjee, 1986; Mukherjee and Pal, 1989) had their origin in his works. The group developed a general time - 
dependent perturbative theory for arbitrarily large time range and generalized to the many - body regime and formulated the first general time-dependent coupled cluster theory for wave functions of arbitrary complexity. Most important contributions came in the form of cumulant based quantum chemistry and statespecific coupled cluster theory for potential energy surfaces (called Mk-MRCC) (Mahapatra et al., 1999) and linear response formulation. Mainly due to his works, India became an important center of coupledcluster theory which was pursued by others directly from his group or inspired by him. Coupled-cluster was used for benchmarking many other approximate methods, including approximate versions of DFT. Following this, active groups on coupled-cluster emerged at NCL Pune ( S Pal, N Vaval), University of Hyderabad (M Durga Prasad), Indian Institute of Astrophysics (B P Das, Rajat Chowdhuri), IISER Mohali (K R Shamasundar) and various other places. The work that emerged from India can be classified into developments of multi-reference versions, mainly Fock space coupled-cluster (FSCC) for various difference energies of spectroscopic interest and the properties of states obtained by linear response of FSCC, state-selective coupled-cluster for potential energy surfaces, relativistic versions of coupled-cluster (from various groups of Mukherjee, Pal, Vaval, Chowdhury and Das), linear response coupled-cluster, alternate variants of coupled-cluster, in particular, extended coupled-cluster and its response. The group of Pal developed recently complex absorbing potential based coupled-cluster method for resonance and decay problems (Sajeev et al., 2014). The group of Das implemented relativistic quantum chemistry, in particular coupled-cluster theory, which was followed by other groups. Durga Prasad's group actively developedcoupled-cluster for the description of molecular vibrations (Prasad, 1988), both the energetics of the stationary states and the dynamics of the non-stationary states at $0 \mathrm{~K}$ as well as at finite temperatures. Shamasundar worked on multireference configuration interaction (MRCI) method based on internally contracted (IC) excitations required to correlate a zeroth-order approximation to the full wave-function. Many in-house codes were written based on the theories developed in India. One of the persons who developed an in-house code based on electrostatic potential is Professor Shridhar Gadre, trained in the school of Professor Narasimhan. Heinvestigated chemical reactivity and weak interactions through the use of molecular electrostatic potentials and momentum densities. His group has also contributed to algorithms and codes for efficiently parallelising quantum chemical ab initio calculations. Recently he has pioneered a technique called the Molecular Tailoring Approach that seeks to calculate accurate one-electron properties for large molecules by breaking down the molecules into fragments and then combining calculations on individual fragments (Sahu et al., 2013). Trained in the school of Professor Mihir Chowdhury, Bhattacharyya at IACS worked on algorithms for optimizations relevant to electronic structure calculations (Nandy et al., 2013). Trained in Professor C N R Rao's school was Professor S Ramasesha (IISc Bangalore), who became one among the most active quantum chemistry researchers in the country. His principal work was on density matrix renormalization group and quantum Monte Caro for large systems (Raghu et al., 2002; Pati et al., 1997). Heworked on nonlinear optical properties of molecules and materials. The theme in his group has been modeling electronic and magnetic properties of molecular and low-dimensional materials.

IIT Bombay had witnessed vigorous pursuit of quantum chemistry, starting with Professor A K Chandra who had a stint at IIT Bombay before moving to IISc, Bangalore. Subsequently, Professor B M Deb and later Professor Debashis Mukherjee joined the Department. After they left, groups of Professor Manoj Mishra and Sambhu Datta actively pursued research in quantum chemistry at IIT Bombay. Datta worked on relativistic quantum chemistry (Datta et al., 1978) in the early days and then moved to description of molecular magnets using quantum chemistry. His work also led to an understanding of photosynthesis processes using quantum chemistry. Mishra, on the other hand, worked on propagator based quantum chemistry and was actively pursuing complex scaled propagator methods for resonance problems. Among others, prominent contributions in quantum chemistry were made by Professor K L Sebastian, at IISc Bengaluru, who then moved to areas on dynamical phenomena of complex systems. 


\section{Computational Quantum Chemistry as Predictive Tools}

As the development of theories grew in India, many theorists started using theories to find insights into the molecular structure and chemical reactivity. These insights have helped to make thesetools predictive and valuable. There are several active groups in India and it has been heartening to see the growth of computational chemistry for various chemical problems, including problems in catalysis, hydrogen storage, environmental chemistry, drug discovery and various other areas of interest in materials and chemistry. The growth is heartening and several students have been trained. One of the successful groups is that of Professor E D Jemmis who studied chemistry of various main group elements, polymorphs of elements and their compounds, etc. His research group extracts insights from the numbers based on overlap of orbitals, perturbation theory and symmetry, and devises transferable models. Significant results have been obtained in understanding the reactions of transition metal organometallics, polyhedral boranes, carboranes, silaboranes, electron counting rules for polycondensation, and structure of boron allotropes, weak H-bond, and electronic structure of threedimensional aromatic compounds. Jemmis mno rules (Jemmis et al., 2001) for polyhedral boranes have found a place in textbooks. He developed a schoolon physical organic chemistry, which resulted in successful translation of computational chemistry based on either density functional theory or wave function based methods. One of the successful schools is that of G N Sastry, who worked in fields of nonbonded interactions, buckybowl chemistry, heteroaromaticity, designing structures that defy conventional bonding patterns, pericyclic reactions, biomolecular modeling and computer aided drug design. In particular, his contributions on cation- $\pi$ interactions and their relevance in chemistry and biology may be noted (Mahadevi and Sastry, 2013). Group of Bharatam Prasad has been focusing on drug discovery by a suite of methods based on DFT and chemoinformatics. Trained originally in the school of Ramasesha, Pati made significant contributions to the understanding of materials using computational chemistry, mainly DFT. His group developed the formalism for computing charge carrier mobility and thermoelectric behavior for systems, studied electronics materials for molecular memory devices, switching devices, photon assisted coherent amplification and destruction of tunneling, catalysis for water splitting, etc. Sunoj at IIT Bombay employs quantum chemical tools of density functional and $a b$ initio theories in the study of complex catalytic reactions. A number of quantum chemical predictions of enantioselective reactions from his group has prompted independent experimental validation by research groups world-wide (Reddi et al., 2019).

\section{DFT in India: Landmark Contributions and Vision}

Besides electronic structure calculations, the adventures and advances in DFT have been realized through a wide variety of other routes. In chemistry particularly, DFT made its entry into the scene through the window of conceptual aspects and played a significant role in providing a rigorous foundation of concepts in chemistry in the mid-seventies and has subsequently given birth to the popular branch known as the "conceptual DFT". Proposed originally as a ground state theory, DFT has been promoted to cover time-dependent situations, excited states, spinpolarized situations, etc. In India, work on DFT has been carried out in almost all directions that are pursued elsewhere and in which it is important. This includes conceptual DFT and foundations of chemical concepts, development of formalisms, time-dependent (TD) DFT, excited states, computational aspects, interpretive aspects, investigation of structure, reactivity and dynamics of atoms, molecules, clusters and solids, unification of TD DFT with quantum fluid dynamics (QFD) to form QFDFT, as a tool for investigating dynamics of collisions, etc. Work on DFT and QFD has been pursued more extensively by several groups in India.

Several people including B M Deb, K D Sen, M S Gopinathan, P K Chattaraj, S K Ghosh, S R Gadre, M K Harbola, S Pal have been involved in the development of theoretical formalism of DFT in some form or the other. There are a large number of persons working in the area of electronic structure calculations using DFT through software such as Gaussian, GAMESSS, etc. There are some directly associated with the conceptual aspects of DFT, which include PK Chattaraj, S Pal, S K Ghosh, T K Ghanty, R K Roy, D C Ghosh, K D Sen and several others. 


\section{Present Scenario in India: Subtopics of Research on DFT in India}

In India there have been many groups working on different aspects and approaches of DFT. In the initial years, there have been emphasis on electron density based interpretive models within the framework of quantum mechanics (QM). The Hellmann-Feynman theorem has been used to calculate the forces on atoms in a molecule, for which only the electron density is necessary and sufficient. This has led to the development of the well known models of molecular geometry through the so called "Force Concept in Chemistry", as proposed by Professor B M Deb (Deb, 1981). This has been generalized and extended to develop the concept of"Internal Stresses in Molecules" in their stationary states (Bamzai and Deb 1981). A classical model was developed for the stability of atoms and molecules based on the concept of balance of electrostatic force on electron cloud by forces of quantum origin, which was linked with the "Bohm potential" which has been known to play an important role in interpretive aspects of QM. Soon it was generalized to cover time-dependent situations, and QFD was developed in 3-D space even for manyelectron systems. DFT has been generalized to cover TD systems and phenomena, in the form of TDDFT for oscillating time-dependence. This provided a formalism to deal with interaction of radiation with matter within DFT and approaches to obtain polarizability (static as well as dynamic) were developed. An amalgamation of TD DFT with QFD was proposed giving birth to QFDFT, which was used to investigate problems involving collision phenomena and interaction of atoms and molecules with an external field including laser field, as extensively investigated by Deb and Chattaraj et al. (1998).

TD DFT has been formulated within a more general set up for many-electron systems subjected to time-dependent electric and magnetic fields, establishing the existence theorem, as well as deriving the single particle equations with effective scalar and vector potentials. This formalism is the most general formulation of TDDFT and has been developed in India (Ghosh and Dhara, 1988). The kinetic and exchange energy functionals for electrons in magnetic field are also derived.

One of the major problems in DFT is the lack of availability of exact expressions for the kinetic and exchange-correlation (XC) energy density functionals (EDF). While kinetic energy (KE) is commonly treated through the Kohn-Sham equations, it introduces the orbital densities instead of the total density as the basic variable. In the orbital-free DFT, the forerunner is the Thomas-Fermi theory, which is limited in scope for atomic and molecular systems, where the electronic density is quite inhomogeneous. Therefore, there was emphasis on developing new $\mathrm{KE}$ functionals that can lead to the shell structure in the radial density of atoms. New routes to better XC functionals have also been investigated. New approaches to the exchange energy functionals for the excited state have been developed by Harbola at IIT-Kanpur. Several aspects of DFT has also been investigated by Amlan Roy at IISER Kolkata.

The work on conceptual aspects of DFT and reactivity theory has been quite prominent in India, and the groups involved are from different Institutes, such as IIT-Kharagpur, NCL, BARC, BITS-Pilani, etc to name a few. The maximum hardness principle proposed earlier (Parr and Chattaraj, 1991) has been verified by considering the geometry changes during molecular vibrations, and other configurations in the process of geometry optimization. Other principles like minimum polarizability principle, electrophilicity equalization principle, etc are also proposed. New concepts of electrophilicity, and several other reactivity indices have also been introduced.

Models for chemical binding have been proposed from time to time by several groups. The chemical potential and hardness generalized to spinpolarized systems within a DFT framework have been used to model covalent binding in homonuclear diatomic systems by the BARC group (Ghanty and Ghosh, 1994). The concepts of bond Chemical potential have also been introduced to account for covalent contribution to chemical binding. Pal's group at NCL and a few others have carried out extensive work on various aspects of chemical reactivity and chemical binding.

\section{Chemical Dynamics}

Alongwith structure, chemical dynamics, a more recent development globally, came into shape in India with efforts from Professor Sathyamurthy (IIT Kanpur and IISER Mohali), who made significant 
contributions in the area of molecular reaction dynamics using accurate potential energy surfaces. His efforts gradually were shaped into an active school of chemical dynamics in the country, investigating the effect of reagent rotation, vibration and orientation on reaction cross section, chaos and fractals in chemical dynamics, reactive scattering resonances in atom-molecule collisions, transition state spectrum, isotope effect on Raman excitation profiles, channel control in chemical reactions and vibration mediated photodissociation. Mahapatra (Univ. of Hyderabad), originally from the school of Professor Sathyamurthy, started in India investigating quantum dynamics for few atomic systems, specially working on nonadiabatic effects on reaction dynamics, multi-mode dynamics, vibronic coupling and excited state dynamics. His group is active in studying dynamics on strongly coupled potential energy surfaces. Professor Sathyamurthy's students, who are active in research on dynamics, include Sanjay Kumar (IIT Madras), Ashwani Tiwari (IISER Kolkata) among others.

Adhikari at IACS Kolkata studied non-adiabatic reaction dynamics beyond Born-Oppenheimer approximation and its applications on spectroscopic and scattering processes. His group also studied and developed methods for multi-electronic multi-mode molecular systems, molecule-surface scattering, role of Jahn-Teller effects, etc. (Sarkar and Adhikari, 2006).

\section{Growth of Computational Chemistry}

In recent years, there are several groups in India working on computational chemistry using both $a b$ initio and DFT methods. Many useful applications on catalysis, gas storage, environment and renewable energy are beginning to emerge. As is the trend, several groups who were working purely on formulations and coding, have also begun to spend some of their efforts on such useful applications. Many of the earlier works described in the article also belong to this domain of computational chemistry. It is impossible to cover the work of all groups in this article. It may be sufficient to say that some of the applications using quantum chemistry are not just by theoreticians, but also by experimentalists. In this article, work mainly on computational chemistry is covered.
Lots of work have been carried out on the chemistry of atomic and molecular clusters, including structure and stability of water clusters (see, e.g., Maheshwary et al., 2001), water under confinement, etc. Subramanian at CLRI Chennai is an active researcher on some of the above applications and has used the methods for gas storage in porous materials, catalysis and electronic applications. Similarly, Rajaraman at IIT Bombay has been active on magnetic properties of materials by computational DFT as well as $a b$ initio wave function based methods. Among others, mention may be made of Suresh at NIIST, Deka at Tezpur University, Ghanty, Maity and Chandrakumar at BARC etc. Several young practitioners of theory are making useful contributions at several IITs, IISERs, central as well as state Universities as well as institutions like BITS Pilani. Almost every institution has theory groups working. It is difficult to enlist all of them. In addition to early generation IITs, e.g. IIT Bombay, IIT Guwahati, IIT Madras, IIT Kanpur, IIT Kharagpur and IIT Delhi, newer IITs are making their mark in quantum chemistry research. IISER Kolkata, Pune, Mohali, Thiruvananthapuram, Bhopal have active groups working on useful chemical problems.

\section{The Road Ahead: The Ideal Scenario}

In India, a lot of wave function based methodological research has taken place. Some of these contributions are in the frontline and pioneering. However, development of codes has been sketchy, mostly confined to in-house development. While there is definite scope of much more formal developments, it is essential to come up with efficient computer codes, both in structure and dynamics, to make global impact in the area. On the other hand, there have been a few methodological developments in DFT. The primary emphasis on research in DFT has been mostly towards application of DFT in the modelling and design of molecules and materials with desired tunable properties. Accordingly, lots of research results involving design and properties of novel materials (bulk as well as confined systems) are being reported. In the area of DFT, India can easily contribute more to the development of efficient fast computer codes. The growth of computational quantum chemistry has been undoubtedly fuelled by general purpose software for molecules and solids available commercially, principally due to the efforts in western world. While such growth 
of the community is certainly welcome, unfortunately it has not been matched by the growth of formulation or more theoretical aspects of quantum chemistry. Indian expertise on development of theoretical formalism should be further strengthened and utilized so as to have opening of new windows leading to appearance of newer routes of theory and applications within the framework of both wave function and DFT. In this area too, the issue of exploiting the Indian expertise in developing computer codes for more efficient and faster computations of electronic structure theory based on DFT exists. Also it may be conjectured that cross fertilization of ideas of wavefunction and DFT based approaches to treat electron correlation might lead to newer developments in this area of research in quantum chemistry.

\section{Concluding Remarks}

In this article, major trends of research in quantum chemistry are covered and as stated in the beginning and the coverage is by no means exhaustive. Only representative list of publications is quoted in this article. For a more exhaustive list of work, websites of researchers and institute web sites may be referred. The accounts are based on personal perceptions of the authors on the status of quantum chemistry in India. Research originating from India is mainly covered. Almost every institution at present has quantum theoreticians as well as computational

\section{References}

Bamzai A S and Deb B M (1981) The role of single-particle density in chemistry Rev Mod Phys 53 95-126

Chattaraj P K, Sengupta S and Poddar A (1998), Quantum fluid density functional theory of time dependent processes Int J Quant Chem 69 279-291

Cizek J (1966) On the Correlation Problem in Atomic and Molecular Systems. Calculation of Wavefunction Components in Ursell-Type Expansion Using Quantum Field Theoretical Methods J Chem Phys 454256

Datta S N, Ewig C S and van Wazer J R (1978) Application of effective potentials to relativistic hartree-fock calculations Chem Phys Letters 57 83-89

Deb B M (1981) The Force Concept in Chemistry, Published by Van Nostrand Reinhold

Deb B M (1984) Current status of theoretical chemistry in India chemists. Several experimentalists are also using computation to design and confirm experimental results. While we have mainly covered the area of quantum chemistry, theories and simulations using classical methods have not been covered at all. However, it is important to mention that the area of combining multiple length scale theories has been well developed in last few decades. In particular, QM/MM (a combination of quantum mechanics with molecular mechanics) has been recognized appropriately for biological systems. There are only a very few groups which are working on $\mathrm{QM} / \mathrm{MM}$, either in terms of development or applications. However, it is hoped that there will be many practitioners using multi-scale simulations for large, complex and extended systems. It is heartening to note that globally the area of quantum chemistry has now emerged as very important in solutions to many chemical and allied problems. India has become one of the major contributors to the growth of the area and will contribute much more hopefully in the near future in the hands of the younger generation of bright quantum chemists. In this context, an earlier article written almost four decades ago will be of interest (Deb 1984).

\section{Acknowledgements}

We acknowledge several sources and literature, including websites of several researchers, for some of the materials presented in this article.

Current Science 53 1061-1068

Fermi E (1927) Un Metodo Statistico per la Determinazione di alcune Prioprietàdell ‘Atomo' Rend Accad Naz Lincei 6 602-607

Ghanty T K and Ghosh S K (1994) Spin-polarized generalization of the concepts of electronegativity and hardness and the description of chemical binding, J Am Chem Soc $1163943-$ 3948

Ghosh S K and Dhara A K (1988) Density-functional theory of many-electron systems subjected to time-dependent electric and magnetic fields Physical Review A 38 11491158

Heitler W and London F (1927) Wechselwirkungneutraler Atome und homöopolare Bindungnach der Quantenmechanik Zeitschriftfür Physik 44 455-472

Hohenberg P and Kohn W (1964) Inhomogeneous Electron Gas 
Physical Review B 136 864-871

James H M and Coolidge A S (1933) The Ground State of the Hydrogen Molecule The Journal of Chemical Physics 1 825

Jemmis E D, Balakrishnarajan M M and Pancharatna P D (2001) A Unifying Electron-Counting Rule for Macropolyhedral Boranes, Metallaboranes, and Metallocenes $J$ Am Chem Soc 123 4313-4323

Kohn W and Sham L J (1965) Self-Consistent Equations Including Exchange and Correlation Effects Physical Review 140 A1133-A1138

Löwdin Per-Olov (1955) Quantum Theory of Many-Particle Systems. III. Extension of the Hartree-Fock Scheme to Include Degenerate Systems and Correlation Effects Physical Review 97 1509-1520

Mahadevi A S and Sastry G N (2013) Cation”ð Interaction: Its Role and Relevance in Chemistry, Biology, and Material Science Chem Rev 113 2100-2138

Mahapatra U S, Datta B and Mukherjee D (1999) A sizeconsistent state-specific multireference coupled cluster theory: formal developments and molecular applications $J$ Chem Phys 1106171

Mukherjee D (1986) Aspects of linked cluster expansion in general model space many body perturbation and coupled cluster theory Int J Quantum Chem 30 S20, 409

Mukherjee D and Pal S (1989) Use of Cluster Expansion Methods in the Open-Shell Correlation Problem Adv Quant Chem 20291

Nandy S, Chaudhury P. and Bhattacharyya S (2002), Stochastic diagonalization of Hamiltonian: A genetic algorithm based approach Intern J Quantum Chem 90 188-194

Nataraj H S, Sahoo B K, Das B Pand Mukherjee D (2008) Intrinsic electric dipole moments of paramagnetic atoms: rubidium and cesium Phys Rev Lett 101033002

Parr R G and Chattaraj P K (1991) Principle of maximum hardness $J$ Am Chem Soc 113 1854-1855

Pati S K, Ranasesha S and Sen D (1997) Low-lying excited states and low-temperature properties of an alternating spin-1- spin-1/2 chain: A density-matrix renormalization-group study Physical Review B 558894

Pauling L (1931) The Nature of the Chemical Bond, Application of results obtained from the quantum mechanic and from a theory of paramagnetic susceptibility to the structure of molecules J Am Chem Soc 53 1367-1400

Prasad M D (1988) A time dependent coupled cluster method: A new approach to the calculation of molecular absorption spectra J Chem Phys $\mathbf{8 8} 7005$

Raghu C, Pati Y A and Ramasesha S (2002) A density matrix renormalization group study of low-lying excitations of polyacene within a Pariser-Parr-Pople model Phys Rev B $\mathbf{6 6} 035116$

Reddi Y, Tsai C -C, Avila C, Toste D F, Sunoj R B (2019) Harnessing Noncovalent Interactions in Dual Catalytic Enantioselective Heck-Matsuda Arylation J Am Chem Soc 141998

Sajeev Y, Ghosh A, Vaval N and Pal S (2014) Coupled cluster methods for autoionization resonances Int Rev Phys Chem 33397

Sahu N, Yeole S D and Gadre S R (2013) Appraisal of Molecular Tailoring Approach for Large Clusters J Chem Phys 138 104101

Sarkar B and Adhikari S (2006) Extended Born-Oppenheimer equation for a three-state system JChem Phys $\mathbf{1 2 4} 074101$

Schroedinger E (1926) Anundulatory theory of the mechanics of atoms and molecules Phys Rev 281049

Sen P N and Basu S (1968) Free-electron model and triplet-state lifetime of benzene Intern J Quantum Chem 2 183-186

Sen K D and Narasimhan P T (1975) Nuclearhexadecapoleanti shielding factors Physical Review 11 1162-1167

Slater J C (1972) Statistical exchange-correlation in the selfconsistent field Advan Quantum Chem 61

Maheshwary S, Patel N, Kulkarni AD and Gadre (2001) Structure and stability of water clusters $\left(\mathrm{H}_{2} \mathrm{O}\right)_{\mathrm{n}}, \mathrm{n}=8-20$ : An ab initio investigation $J$ Phys Chem A 105 10525-10537

Thomas L H (1927) The calculation of atomic fields Proc Camb Phil Soc 23 542-548. 